Revista ELectrónica de Investigación y EValuación Educativa
RELIEVE

ISSN: 1134-4032
e-Journal of Educational Research, Assessment and Evaluation

\title{
PISA y TALIS ¿congruencia o discrepancia?
}

\section{PISA and TALIS, congruence or discrepancy?}

\section{Fernández-Díaz, María-José; Rodríguez-Mantilla Jesús-Miguel \& Martínez-Zarzuelo, Angélica} Universidad Complutense de Madrid; Facultad de Educación. (Spain)

\begin{abstract}
International educational evaluations on a grand scale provide information of interest to the educational, scientific and political community. Many studies have derived from them, both nationally and internationally. On the other hand, it is well known from the beginning of these macro-assessments the diversity of opinions they have generated. In this context, the aim of the study presented here is to analyse the relationships between the results from PISA 2012 and those relating to teaching practice of secondary TALIS 2013, trying to find out the consistencies and discrepancies between the results of both. Data from TALIS-PISA link have been used. The descriptive analysis carried out taking the overall scores on both evaluations show obvious discrepancies, as countries occupying the top positions in the results of students in PISA are located in the last places in teaching practice of teachers. The analysis also show the lack of the expected coherence. These results lead to reflect on the possible underlying causes and so it is proposed the need for a coordinated design of both evaluation processes
\end{abstract}

Reception Date
2016 April 7

Approval Date

2016 June 26

Publication Date:

2016 June 26

\section{Keywords:}

TALIS, PISA, OECD, educational assessment, international evaluations, academic achievement, teaching practice, teaching staff, research, compulsory secondary education

\section{Resumen}

Las evaluaciones educativas internacionales a gran escala ofrecen información de interés para la comunidad educativa, científica y política. Son muchos los estudios derivados de ellas, tanto a nivel nacional como internacional. Por otra parte, es bien conocida, desde el origen de estas macroevaluaciones, la diversidad de valoraciones que han generado. En este contexto, el objetivo del trabajo que aquí se presenta es estudiar las relaciones entre los resultados de PISA 2012 y los relativos a práctica docente de secundaria de TALIS 2013, tratando de analizar las congruencias o discrepancias entre los resultados de ambas. Se tomaron los datos del estudio TALIS-PISA link. Los análisis realizados, de tipo descriptivo, tomando las puntuaciones globales en ambas evaluaciones muestran discrepancias evidentes, ya que países que ocupan primeras posiciones en los resultados de los estudiantes en PISA se ubican en últimos lugares en práctica docente del profesorado., donde se supone que las relaciones se debieran producir con mayor intensidad al existir una relación más directa entre los alumnos y los profesores evaluados. Los análisis realizados muestran igualmente la falta de coherencia esperada. Estos resultados, llevan a reflexionar sobre las posibles causas que subyacen y se propone la

Fecha de recepción 7 Abril 2016

\section{Fecha de} aprobación 26 Junio 2016

Fecha de publicación 26 Junio 2016 necesidad de un diseño coordinado de ambos procesos evaluativos.

\section{Palabras clave:}

TALIS, PISA, OCDE, evaluación educativa, evaluaciones internacionales, rendimiento académico, práctica docente, profesorado, investigación, Educación Secundaria Obligatoria

Las publicaciones que se van generando sobre estas dos evaluaciones internacionales reflejan el interés de la comunidad académica y profesional de una mayoría de países por estos estudios. Indudablemente desatan posiciones bien diversas en el mundo de la educación, desde los defensores a ultranza hasta aquellos detractores que lanzan críticas

\section{Autor de contacto / Corresponding author}

Fernández-Díaz, María-José. Facultad de Educación. C/Rector Royo Villanova, s/n, 28040-Madrid (España)._mjfdiaz@edu.ucm.es. 
relevantes desde posiciones distintas (Mendizábal, 2016; Carabaña, 2015; Sjøberg, 2015; Sánchez \& Delgado, 2013; Ferrer, 2012; Pérez \& Soto, 2011; Ruiz, Gil, Navas, Ramos, Ruiz \& Núñez, 2011; Font, Badia, Alemany, Besora, Gisbert, Arce, Alonso, Seuba, Castilla, Lamo, Valdivia, Villanueva \& Boekaerts, 2009; Grek, 2009; Martín \& Rizo, 2009; Mortimore, 2009; Neves, 2008; Dohn, 2007; Hernández, 2006; Sánchez \& García-Rodicio, 2006).

Es indudable que estas macroevaluaciones o evaluaciones a gran escala aportan información relevante y permiten realizar estudios que sobrepasan el ámbito localista más reducido y con menor representatividad, incluso cuando se realizan con muestras a nivel de país, el modo de hacer más común en el ámbito educativo. Sin duda, esta visión internacional ofrece posibilidades impensables, información que permite realizar comparaciones y detectar factores asociados a las diferencias entre los países. Sin embargo, en muchos casos, sus interpretaciones se utilizan con objetivos y fines para los que no fueron ni diseñados ni concebidos, tanto por parte de los adeptos como de los opositores. Discrepancias y argumentos de tipo ideológico, político, económico, y un buen número de razones diversas que permiten valorar o rechazar estas evaluaciones.

Pero no es nuestro objetivo ahondar en estas argumentaciones de unos y otros, sino más bien tratar de analizar las relaciones entre dos de las evaluaciones más importantes auspiciadas por la OCDE (Organization for Economic Co-operation and Development) que, por su naturaleza, parece que debieran evidenciar algún tipo de relaciones. Así, es bien conocido en el mundo de la educación, PISA (Programme for International Student Assessment), cuya primera evaluación se realiza en el año 2000, aplicándose periódicamente cada tres años. Aunque se centra en tres áreas de evaluación: lectura, matemáticas y ciencias, en cada una de las ediciones de PISA, una de ellas siempre se analiza con mayor profundidad que las otras dos. Así, lectura fue el área principal en las ediciones de 2000 y 2009, ciencias en 2006 y matemáticas en 2003 y 2012.

Uno de los objetivos del estudio PISA es tratar de contribuir a la evaluación de lo que saben y son capaces de hacer los jóvenes de quince años de edad. Este estudio no solamente pretende evaluar lo que los jóvenes de esa edad han aprendido en el ámbito escolar, sino también su aprendizaje en otros ámbitos formales e informales. Todo ello desde un punto de vista muy aplicado y contextualizado en el propio entorno del alumno.

Se dirige a evaluar competencias que el estudiante de secundaria ha desarrollado a lo largo de su vida, mostrando los comportamientos o reacciones ante las situaciones de la vida diaria. Es evidente que una parte importante de su influencia viene determinada por las experiencias que ha ido adquiriendo en su trayectoria escolar, conocimientos, actitudes, competencias, etc. que, integradas con las experiencias vividas en otros ámbitos, como la familia, los amigos, las relaciones sociales con personas diferentes, etc. van configurando una forma de reaccionar ante los estímulos que constituyen la prueba de PISA. Sin duda, vienen influenciadas por las vivencias en diversos ámbitos y contextos pero, teniendo en cuenta que las pruebas se aplican en el nivel de secundaria, es lógico pensar que el estudiante ha pasado una gran parte de su tiempo en el ámbito escolar y su influencia es relevante por los propios objetivos de los centros educativos.

Todas las ediciones de PISA han generado una gran cantidad de literatura $y$ publicaciones, tanto a nivel nacional como internacional. Respecto a la última edición de 2012, el MECD (Ministerio de Educación, Cultura y Deporte) y la OCDE han realizado distintas publicaciones (MECD, 2014a, 2014b; 2014c; OECD, 2014a; 2014b, 2014c, 2013a, 2013b, 2013c). Diversos autores han estudiado además diferentes aspectos y contextos de esta edición de PISA (Stacey, 2015; Kelly, Nord, Jenkins, Chan \& Kastberg, 2013; Sedghi, 
Arnett \& Chalabi, 2013; Thomson, De Bortoli \& Buckley, 2013; Villar, 2013; Wheater, 2013; Calero \& Escardíbul, 2012; Peña-López, 2012).

Por otra parte, la evaluación de TALIS (Teaching and Learning International Survey) también promovida por la OCDE, tiene orígenes más recientes. Se trata de un estudio internacional de carácter cíclico que tiene lugar cada cinco años, cuyo objetivo principal es proporcionar información oportuna, comparable y útil para ayudar en los países en la revisión y definición de políticas educativas para el desarrollo de una docencia de alta calidad. Las valoraciones se realizan por parte de directores y profesores de centros con sendos cuestionarios sobre la situación educativa, la profesión docente, la eficacia docente, el clima y la satisfacción laboral, entre otros aspectos. Es un sistema de autoevaluación, con las limitaciones que este sistema conlleva, pero, sin duda, ofrecen información sobre aspectos importantes de los procesos de enseñanza aprendizaje que deben utilizarse para reflexionar sobre la situación de cada país y, en consecuencia, establecer políticas que permitan mejorar la educación a nivel de cada país en particular y a nivel internacional. Hasta el momento se han realizado dos ediciones, TALIS 2008 y TALIS 2013. Con los datos se han realizado numerosos estudios y se han deducido otras tantas conclusiones de interés para la comunidad científica (Gumus \& Bellibas, 2016; Perry, Sealy \& Hawkins, 2016; Albergaria-Almeida, da Silva Lopes \& Martinho, 2015; Lizasoain, Tourón \& Sobrino, 2015; Ming-ren, 2015; MECD, 2014d; OECD, 2014d; Jensen, Sandoval, Knoll \& González, 2012; Vieluf, Kaplan, Klieme \& Bayer, 2012; ME 2009; OECD, 2009).

Tanto la primera como la segunda edición se han centrado en la etapa educativa de Educación Secundaria Obligatoria (Nivel 2, según la International Standard Classification of Education). Sin embargo, también se ofrecieron otras opciones internacionales como Educación Primaria (Nivel 1) y Educación
Secundaria Superior (Nivel 3). En la segunda edición de este estudio, TALIS 2013, también se ha dado la opción de participar en una modalidad, TALIS-PISA Link, que relaciona a nivel de centro la última edición de TALIS y la quinta de PISA, es decir, la correspondiente a 2012. Este estudio ha permitido vincular información proporcionada por alumnos, profesores y directores sobre distintos factores relacionados con la enseñanza y el aprendizaje. Así, entre otros aspectos, ha permitido conocer en mayor profundidad la relación entre el rendimiento académico de los alumnos en distintas competencias y las actitudes y prácticas educativas de los docentes que imparten clase en un mismo centro educativo.

Ahora bien, es evidente que se precisa avanzar en estos estudios, tratando de vincular los datos de PISA con los de TALIS, tomando a estudiantes y profesores de las distintas áreas para poder establecer relaciones que, desde planteamientos teóricos, debieran producirse, $\mathrm{y}$ que los estudios empíricos debieran poner de manifiesto. Como expresa Marina (2013 p.4) "el hecho de que este estudio sea independiente del de PISA resulta escandaloso a un profesional de la docencia”.

Diversos autores han analizado la relación existente entre la formación del profesorado, sus prácticas y los logros de los alumnos (Clotfelte, Ladd \& Vidgor, 2007; Gustafsson, 2003; Wayne \& Youngs, 2003; Wenglinsky, 2002), incluso tomando los resultados de las evaluaciones TALIS 2008 y PISA 2009 (Kaplan \& Turner, 2012). Con esta edición de TALIS 2013 también se ha realizado alguna investigación basada en la relación entre profesores y alumnado. Se encuentran estudios como el de Eveleigh \& Freeman (2012) donde los autores proponen análisis exploratorios de tipo correlacional, el de Sealy, Perry \& DeNicola (2016) en el que se analizan, entre otros aspectos, las relaciones existentes entre la satisfacción laboral y el rendimiento del alumnado, o como el de Méndez (2015), quien realiza un análisis del efecto de las distintas prácticas docentes en el rendimiento de sus 
alumnos. Sin embargo, aunque todos ellos se basan en el análisis de diferentes relaciones entre ambas evaluaciones internacionales, ninguno de ellos lo hace en la línea que aquí se presenta.

Parece obvio que si los estudiantes evaluados muestran resultados influenciados en gran parte por el sistema educativo, las escuelas y los profesores, deben estar relacionados con los mejores sistemas, las mejores escuelas y los mejores profesores. Ante los tímidos intentos que se han realizado con los estudios de TALIS-PISA link y tratando de valorar los resultados de las evaluaciones realizadas en PISA 2012 y TALIS 2013, este trabajo pretende analizar, con la profundidad que la información permite hasta el momento, si los resultados obtenidos en ambas evaluaciones reflejan congruencias o discrepancias entre las valoraciones de la práctica docente de los profesores en TALIS 2013 y los resultados obtenidos por los estudiantes en PISA 2012, planteando algunos interrogantes derivados de ellos que permitan reflexionar y avanzar en estos procesos.

\section{MÉTODO}

\section{Diseño}

El diseño implicado en este estudio es no experimental, de carácter exploratorio, enmarcado, concretamente, dentro de los estudios denominados ex-post-facto.

\section{Población y muestra}

La población de estudio de PISA 2012 estuvo configurada por 13.142.800 alumnos de $2^{\circ}$ de Educación Secundaria Obligatoria (ESO), correspondiente a 34 países $^{1}$. La

\footnotetext{
${ }^{1}$ Países participantes en PISA 2012: Albania, Alemania, Argentina, Australia, Austria, Bélgica, Brasil, Bulgaria, Canadá, Catar, Chile, China (Hong-Kong, Macao, Shanghai, Taipéi), Chipre, Colombia, Corea del Sur, Costa Rica, Croacia, Dinamarca, Emiratos Árabes Unidos, Eslovaquia, Eslovenia, España, Estados Unidos, Estonia, Federación Rusa, Finlandia, Francia, Grecia, Hungría, Indonesia, Irlanda, Islandia, Israel, Italia, Japón, Jordania, Kazajistán, Letonia, Liechtenstein, Lituania, Luxemburgo, Malasia, México, Montenegro, Noruega, Nueva Zelanda, Países Bajos, Perú, Polonia, Portugal, Reino Unido, República Checa, Rumanía, Serbia, Singapur, Suecia, Suiza, Tailandia, Túnez, Turquía, Uruguay y Vietnam.
}

muestra final quedó configurada por 295.416 alumnos de ESO de dichos países.

La población de estudio de TALIS 2013 quedó configurada por 2.835.184 docentes de ESO correspondientes a un total de 34 países $^{2}$. De esta población y tras depurar la base de datos, eliminando los correspondientes a Estados Unidos y Chipre por indicación de la OCDE, se obtuvo una muestra final de 103.862 profesores correspondientes a 32 países participantes. De esta muestra fueron 27.022 profesores los que formaron parte también del estudio TALIS-PISA Link, docentes pertenecientes a un total de 8 países ${ }^{3}$. Esta submuestra está formada por profesores de centros educativos que también participaron en PISA de 2012.

\section{Instrumento}

En el caso de PISA 2012, la prueba empleada por la OCDE consta de 3 grandes unidades de evaluación: Matemáticas, Lectura y Ciencias. Las preguntas en cada área son de opción múltiple y de respuesta abierta. Si bien el desarrollo y descripción detallada de estas pruebas escapan al objetivo de este trabajo, se remite a la información aportada por la OCDE en cualquiera de sus publicaciones (OECD, 2014a, 2014b, 2014c, 2014d, 2013a, 2013b, 2013c, 2013d). Para el presente estudio, utilizaremos como referencia las puntuaciones finales obtenidas en cada una de las áreas, así como en cada una de las tres materias evaluadas.

De los instrumentos empleados por la OCDE en TALIS 2013, un cuestionario para directores y otro para profesores, se utilizó para el presente estudio éste último, tomando los ítems relativos a la práctica docente.

\footnotetext{
2 Países participantes en TALIS 2013: Australia, Brasil, Bulgaria, Chile, Corea, Chipre, Croacia, Dinamarca, Eslovaquia, España, Estados Unidos, Estonia, Finlandia, Francia, Islandia, Israel, Italia, Japón, Letonia, Malasia, México, Noruega, Países Bajos, Polonia, Portugal, República Checa, Rumanía, Serbia, Singapur, Suecia, Bélgica (Flandes), Canadá (Alberta), Emiratos Árabes Unidos (Abu Dabi) y Reino Unido (Inglaterra).

${ }^{3}$ Países participantes en la opción TALIS-PISA link: Australia, España, Finlandia, Letonia, México, Portugal, Rumanía y Singapur.
} 
Concretamente, se utilizaron 38 ítems del conjunto total que configura el cuestionario de TALIS, donde cada profesor debía valorar cada uno de ellos en una escala tipo Likert de 4 y 6 grados, según el ítem.

Dichos ítems se organizan en las siguientes dimensiones (Fernández-Díaz, RodríguezMantilla \& Martínez-Zarzuelo, 2015): Colaboración y coordinación profesional (0-40 puntos. 8 ítems), Metodología docente (0-33 puntos. 11 ítems), Evaluación (0-21 puntos. 7 ítems), Motivación hacia el alumnado (0-12 puntos. 4 ítems) y Control del clima de aula (0-24 puntos. 8 ítems). De igual modo, se creó la variable Ítem Total, correspondiente a la puntuación global del instrumento (en una escala de 0 a 130)

La calidad psicométrica de los instrumentos de PISA, según OECD (2013d), muestran un nivel de fiabilidad excelente para las subescalas de PISA 2012 ( $\alpha$ de Cronbach $=$ 0,914, 0,888 y 0,885 para Matemáticas, Lectura y Ciencias, respectivamente). Para el conjunto de los 38 ítems del instrumento de TALIS 2013 se realizó un estudio de fiabilidad, obteniendo también un valor excelente $(\alpha$ de Cronbach $=0,867)$, no encontrando valores fuera de lo esperado en los índices de homogeneidad de los ítems, por debajo de 0,2 según Hair, Anderson, Tathan \& Black (2009).

\section{Análisis de datos e interpretación de resultados}

Con el objeto de analizar la congruencia o discrepancia entre los resultados de PISA 2012 y TALIS 2013 se realizaron diversos estudios estadísticos utilizando el software SPSS 22.

En primer lugar, se analizaron los datos a nivel global, de los 32 países, tanto en PISA 2012 como en TALIS 2013, con el fin de identificar las posibles relaciones entre las puntuaciones de ambas evaluaciones.

Posteriormente, tomando la muestra de los 8 países participantes en TALIS-PISA Link, se analizaron y compararon las puntuaciones globales en TALIS 2013 y de cada una de sus dimensiones, con las puntuaciones globales en PISA 2012, específicamente en Matemáticas. Con este grupo de países se realizó un estudio descriptivo, representado gráficamente, que pretende mostrar los profesores con distinto nivel docente (alto, medio y bajo) en cada país, que permite ilustrar coherencias o discrepancias dignas de reseñar.

Por último, con el fin de estudiar empíricamente las relaciones entre los dos grupos de puntuaciones, se realizó un análisis correlacional simple, tomando las puntuaciones de Matemáticas en PISA 2012 y las puntuaciones en cada una de las dimensiones de TALIS 2013, además de la puntuación global. De forma complementaria, se realizó un estudio de análisis de varianza (ANOVA y sus contrastes posteriores de Scheffé) tratando de probar las posibles diferencias en Matemáticas en función de los distintos niveles de práctica docente, de acuerdo a las tres categorías definidas anteriormente.

\section{Análisis global de las puntuaciones en TALIS 2013 y PISA 2012: aproximación al estudio de la congruencia o discrepancia.}

Con el objetivo de conocer las puntuaciones obtenidas por los 32 países en TALIS 2013 y PISA 2012 y analizar sus resultados tratando de buscar las posibles relaciones de coherencia o discrepancia entre ambas pruebas, se presentan estudios descriptivos de cada uno de ellos.

Para la configuración de la Tabla 1 se han utilizado las puntuaciones de Ítem Total en TALIS 2013 de cada uno de los países participantes, ordenando los mismos de mayor a menor puntuación obtenida. Igualmente, se han ordenado los países por su puntuación global obtenida en PISA 2012 (gráficamente, mediante flechas, se muestra la relación inversa de las puntuaciones en TALIS 2013 y PISA 2012 de algunos países). 
Tabla 1: Tabla comparativa de los 32 países ordenados según sus resultados en TALIS 2013 y PISA $2012^{4}$

\begin{tabular}{|c|c|c|c|c|c|}
\hline & $\begin{array}{l}\text { TOTAL } \\
\text { TALIS }\end{array}$ & Posición & Posición & $\begin{array}{c}\text { TOTAL } \\
\text { PISA }\end{array}$ & \\
\hline E.A.U. (Abu Dabi) & 82,4 & 1 & 1 & 555,7 & Singapur \\
\hline Portugal & 78,2 & 2 & 2 & 542,5 & Corea \\
\hline Dinamarca & 77,7 & 3 & 3 & 540,4 & Japón \\
\hline Australia & 76,6 & 4 & 4 & 529,4 & Finlandia \\
\hline Rumanía & 76,6 & 5 & 5 & 526,1 & Estonia \\
\hline R.U. (Inglaterra) & 76,3 & 6 & 6 & 522,2 & Canadá \\
\hline Bulgaria & 76 & 7 & 7 & 520,5 & Polonia \\
\hline México & 75,7 & 8 & 8 & 518,8 & Países Bajos \\
\hline Italia & 73,6 & 9 & 9 & 512,5 & Australia \\
\hline Polonia & 73,5 & 10 & 10 & 509,3 & Bélgica (Flandes) \\
\hline Singapur & 73,3 & 11 & 11 & 502,5 & R.U. (Inglaterra) \\
\hline Canadá & 72,7 & 12 & 12 & 500,1 & República Checa \\
\hline Serbia & 71,8 & 13 & 13 & 499,8 & Francia \\
\hline Eslovaquia & 70,7 & 14 & 14 & 498,2 & Dinamarca \\
\hline Letonia & 70,6 & 15 & 15 & 495,9 & Noruega \\
\hline República Checa & 69,8 & 16 & 16 & 493,8 & Letonia \\
\hline Israel & 69,7 & 17 & 17 & 489,6 & España \\
\hline España & 69,4 & 18 & 18 & 489,5 & Italia \\
\hline Chile & 69,1 & 19 & 19 & 488 & Portugal \\
\hline Noruega & 69,1 & 20 & 20 & 484,5 & Islandia \\
\hline Brasil & 68,2 & 21 & 21 & 482,4 & Croacia \\
\hline Suecia & 67,8 & 22 & 22 & 482,1 & Suecia \\
\hline Estonia & 67,8 & 23 & 23 & 474,1 & Israel \\
\hline Francia & 67,4 & 24 & 24 & 471,9 & Eslovaquia \\
\hline Malasia & 67,1 & 25 & 25 & 446,6 & Serbia \\
\hline Bélgica (Flandes) & 66,1 & 26 & 26 & 441,4 & E.A.U. (Abu Dabi) \\
\hline Croacia & 65,5 & 27 & 27 & 440,4 & Bulgaria \\
\hline Finlandia & 64,9 & 28 & 28 & 440,3 & Rumanía \\
\hline Países Bajos & 64,1 & 29 & 29 & 436,3 & Chile \\
\hline Islandia & 63 & 30 & 30 & 417,3 & México \\
\hline Japón & 59,8 & 31 & 31 & 412,7 & Malasia \\
\hline Corea & 57,1 & 32 & 32 & 398,9 & Brasil \\
\hline
\end{tabular}

\footnotetext{
${ }^{4}$ Los países sombreados corresponden a los países participantes en el estudio TALIS-PISA Link
} 
Analizando y comparando las puntuaciones obtenidas por los países en PISA 2012 con TALIS 2013, se observa que países con altas puntuaciones en TALIS 2013, en muchos casos, presentan puntuaciones no congruentes en PISA 2012. Es el caso de Corea, Japón, Finlandia e incluso Países Bajos, con puntuaciones muy bajas en TALIS 2013, pero con altas o muy altas en PISA 2012. Esta discrepancia entre resultados, se encuentra también en países como Emiratos Árabes Unidos (Abu Dabi), Portugal, Rumanía, Bulgaria o México, que obtienen puntuaciones altas en TALIS 2013, pero promedios bajos o muy bajos en PISA 2012. Únicamente en el caso de países como España, Letonia o Suecia encontramos cierta congruencia entre los resultados obtenidos en ambas pruebas.

Cabe señalar que, en términos generales, los países con mayores puntuaciones en el Ítem Total de TALIS 2013 presentan altas puntuaciones en las diferentes dimensiones evaluadas. En este sentido, si bien un estudio descriptivo más pormenorizado en esta línea de análisis (relación entre puntuaciones en las Dimensiones y el Ítem Total) resulta interesante, supera el objetivo del presente trabajo. No obstante, se muestra en el Anexo final la descripción detallada por países en cada dimensión.

\section{Análisis de congruencias o discrepancias en TALIS-PISA Link}

Con el fin de profundizar un poco más en estas congruencias o discrepancias, se ha tomado únicamente para este estudio una parte de la muestra global, correspondiente a los participantes en el estudio TALIS-PISA Link, configurada por 8 países de los que se dispone información de alumnos y profesores de Matemáticas de Secundaria de cada centro que participaron en TALIS 2013 y PISA 2012, por lo que cabría esperar una mayor relación entre los resultados de ambas pruebas. Si bien desafortunadamente no se puede garantizar que todos los profesores evaluados lo hayan sido de los alumnos evaluados de cada centro en PISA, esta circunstancia sí se ha podido producir en algunos casos, siendo además comprensible que el equipo de profesores ha podido utilizar metodologías similares con los alumnos del mismo nivel educativo $\mathrm{y}$ en relación con el Proyecto Curricular de cada centro. Recordemos que los países participantes en TALIS-PISA Link son: Australia, España, Finlandia, Letonia, México, Portugal, Rumanía y Singapur.

En primer lugar, se han realizado estudios por dimensiones en TALIS 2013 y en Matemáticas (PISA 2012), tratando de relacionar los resultados.

Tabla 2: Estudios descriptivos de los 8 países participantes en TALIS-PISA Link ordenados por la puntuación global obtenida en el Ítem Total de TALIS 2013, junto con la media global y de matemáticas en PISA 2012

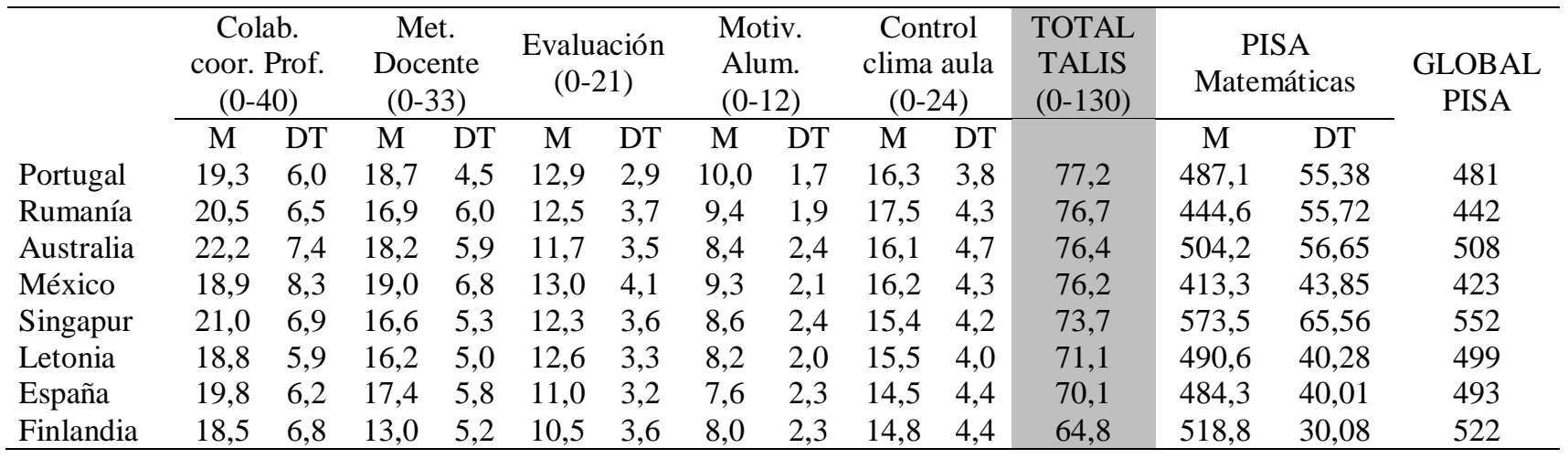

En la Tabla 2 se observa que países como Portugal, Rumanía y México presentan puntuaciones altas y medio-altas en el Ítem
Total de TALIS 2013, así como en Metodología docente, Motivación hacia los alumnos y en Control del clima del aula. No 
obstante, al igual que en el estudio global del conjunto de los 32 países, se observa que los países que presentan puntuaciones globales altas en TALIS 2013 presentan niveles en Matemáticas (y en PISA 2012 global) no siempre congruentes. Así, por ejemplo, países como Portugal, Australia y México presentan puntuaciones altas en TALIS, pero bajas en Matemáticas. En otros casos, como en
Finlandia, obtienen puntuaciones muy bajas en TALIS y muy altas en Matemáticas.

Desagregando la información de los resultados de TALIS 2013 y categorizando en 3 niveles el desempeño docente (nivel Alto, Medio y Bajo), en la Figura 1 se observa cómo países con altas puntuaciones en PISA 2012, como Finlandia, presenta un mayor porcentaje de profesores con Bajo nivel en desempeño docente.

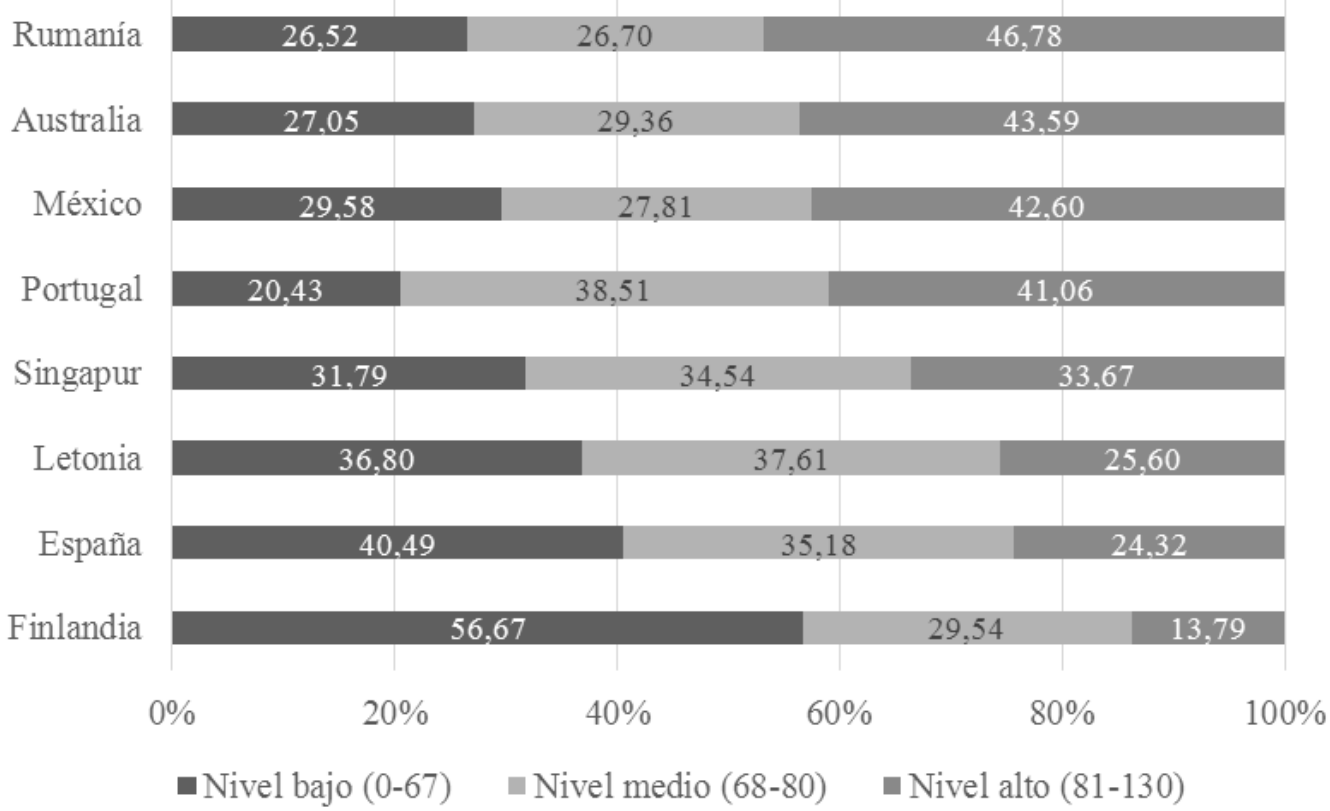

Figura 1: Porcentajes de profesores con alto, medio y bajo nivel en desempeño docente en países de TALIS-PISA Link.

De igual modo, países como Rumanía y México presentan altos porcentajes (por encima del 42\%) de profesores con Alto desempeño docente, cuando sus puntuaciones en PISA 2012 les sitúan en últimas posiciones. Por su parte, Portugal presenta porcentajes equiparados de profesores con Alto y Medio nivel docente, mientras que Singapur y Letonia presentan porcentajes muy equilibrados de profesores con Bajo, Medio y Alto desempeño docente, $\mathrm{y}$, sin embargo, sus puntuaciones en PISA 2012 no resultan congruentes (Singapur, por ejemplo, ocupa la primera posición en PISA 2012 y en Matemáticas, mientras que Letonia se encuentra en una posición intermedia).

\section{Análisis Correlacional de los países participantes en TALIS-PISA Link}

Tratando de contribuir al objetivo del trabajo desde distintas técnicas de análisis, se presenta a continuación un estudio correlacional entre los resultados de los países de TALIS-PISA Link.

Las correlaciones de Pearson obtenidas entre las dimensiones de TALIS 2013 y en Matemáticas PISA 2012 indican la no existencia de correlación (ver Tabla 3), si bien por la magnitud de la muestra resulte que dichas correlaciones son, en casi todos los casos, significativas al nivel de significación de 0,01 (a excepción de $\mathrm{r}_{\text {Matemáticas-Colaboración) }}$ 
aunque con valores prácticamente irrelevantes (cercanos a cero). Los resultados son coherentes con los obtenidos en los análisis anteriores. Incluso, llama la atención cómo en el caso de la correlación entre Matemáticas (PISA 2012) con Evaluación, Motivación hacia el alumnado, Control de clima de aula y el Ítem Total, aunque bajas, son negativas, reflejando una relación inversa $y$, en consecuencia, mostrando la discrepancia a la que ya se ha aludido.

Tabla 3: Correlación de Pearson entre las dimensiones TALIS 2013 con los resultados en Matemáticas PISA 2012 (submuestra de países participantes en TALIS-PISA Link)

\begin{tabular}{|c|c|c|c|c|c|c|c|}
\hline & & $\begin{array}{l}\text { Colab. } \\
\text { Prof. }\end{array}$ & $\begin{array}{l}\text { Met. } \\
\text { Doc. }\end{array}$ & Evalua. & $\begin{array}{l}\text { Mot. } \\
\text { Alum. }\end{array}$ & $\begin{array}{l}\text { Ctrl. clima } \\
\text { aula }\end{array}$ & $\begin{array}{c}\text { Ítem Total } \\
\text { TALIS }\end{array}$ \\
\hline \multirow[t]{2}{*}{ PISA MATEM. } & Pearson & 0,009 & $-0,064$ & $-0,03$ & $-0,027$ & 0,017 & $-0,025$ \\
\hline & Sig. & 0,146 & 0,000 & 0,000 & 0,000 & 0,007 & 0,000 \\
\hline
\end{tabular}

\section{Análisis diferencial en el rendimiento en Matemáticas en función del nivel de desempeño docente}

Para ilustrar con más evidencias los resultados que se han obtenido hasta el momento, retomamos las categorías en que se han clasificado el conjunto de los profesores de los 8 países según las puntuaciones en
TALIS 2013 (nivel Alto, Medio y Bajo desempeño docente). Se realizó un análisis de varianza (ANOVA y los contrastes posteriores de Scheffé) para comprobar las diferencias en los resultados en Matemáticas (PISA 2012) en función del nivel de práctica docente, tomando las 3 categorías señaladas.

Tabla 4: ANOVA de un factor para los resultados en Matemáticas PISA 2012 en función del nivel de desempeño docente de los profesores categorizado en Bajo, Medio y Alto según TALIS 2013.

\begin{tabular}{lclllllc}
\hline & & N & Media & DT & F & Sig. & $\eta^{2}$ \\
\hline Matemáticas & (PISANivel bajo (0-67) & 9068 & 495,44 & 61,47 & 17,79 &, 000 &, 001 \\
2012) & Nivel medio (68-80) & 8614 & 496,24 & 64,12 & & & \\
& Nivel alto (81-130) & 8517 & 490,79 & 68,53 & & & \\
\hline
\end{tabular}

Como se puede observar (Tabla 4), las medias en Matemáticas presentan escasas diferencias entre los niveles de práctica docente (promedios entre 490,79 y 496,24). Sin embargo, las diferencias son significativas $(\mathrm{p}<0,01)$ por la magnitud de la muestra -como ya se ha señalado anteriormente-, pero irrelevantes, tal y como indica el bajo valor del tamaño del efecto $\left(\eta^{2}=0,001\right)$. Sorprende especialmente cómo los profesores de nivel alto presentan la más baja puntuación en PISA, evidencia de lo que se viene comprobando en los análisis anteriores, es decir, de la discrepancia en los resultados entre TALIS y PISA.

Se han realizado contrastes posteriores de Scheffé, cuyos resultados se muestran en la siguiente tabla:

Tabla 5: Contrastes posteriores de Scheffé para los resultados en Matemáticas PISA 2012 en función del nivel de desempeño docente de los profesores categorizado en Bajo, Medio y Alto según TALIS 2013

$$
\text { Dif. de medias Error típico Sig. }
$$

\begin{tabular}{llcrr}
\hline Nivel medio (68-80) & Nivel bajo (0-67) &, 80306 &, 97354 &, 712 \\
\hline Nivel alto (81-130) & Nivel bajo (0-67) & $-4,65115^{*}$ &, 97638 &, 000 \\
& Nivel medio (68-80) & $-5,45422^{*}$ &, 98876 &, 000 \\
& Nivel medio (68-80) & $-8,56893^{*}$ &, 95621 &, 000 \\
\hline
\end{tabular}


Como se puede observar, se encuentran diferencias significativas en Matemáticas entre los profesores de nivel Alto en desempeño docente respecto a los nivel Medio y Bajo (Tabla 5). No obstante, no se encuentran diferencias significativas en Matemáticas en PISA 2012 entre profesores de niveles Bajos y Medios en desempeño docente.

\section{Conclusiones y discusión}

Como se ha señalado, el objetivo del trabajo era intentar analizar y profundizar en las relaciones entre resultados en dos evaluaciones internacionales que, desde la teoría, debieran tener alguna relación, rendimiento de estudiantes en PISA y valoraciones de los profesores en TALIS del mismo nivel educativo, es decir Secundaria. En síntesis, ¿los datos muestran coherencias o discrepancias?

En primer lugar, hay que destacar la escasa investigación realizada en esta línea que debiera fundamentar en gran parte estos estudios y la necesidad de vincular las evaluaciones de TALIS con las de PISA, desde su diseño, para poder profundizar en los datos y resultados, y extraer conclusiones relevantes y consistentes para reflexionar y realizar los cambios que procedan en los distintos países, en sus políticas, sus sistemas educativos, la formación de su profesorado, entre otros elementos. Ello podría posibilitar conclusiones de más calado a las que nos debieran llevar este tipo de evaluaciones, sin menospreciar en ningún caso la importancia de las realizadas hasta el momento, a gran escala, que han supuesto un punto de inflexión en las evaluaciones parciales, de carácter localista, realizadas con muestras pequeñas y con dudosa representatividad, en muchos casos. Los datos extraídos de estas evaluaciones están generando estudios, investigaciones, análisis comparativos y, en algunos casos, cambios en las políticas educativas de ciertos países que miran a aquellos que, en general, mantienen consistentemente posiciones altas a lo largo de las evaluaciones que se han realizado hasta el momento. Pero es obvio que hay que avanzar en una mejora de estos procesos, ya que forma parte intrínseca de una evaluación dinámica que progresa y avanza para extraer lo mejor y producir un cambio sustentado en evidencias.

Los resultados de los diversos análisis realizados, dentro de las limitaciones ya señaladas de las bases de datos disponibles, apuntan algunas reflexiones e interrogantes de interés.

Como se ha podido apreciar, los análisis muestran con claridad la falta de congruencia entre los resultados de los distintos países obtenidos en PISA 2012 y TALIS 2013, produciéndose en muchos casos incongruencias notables. Así, países que, consistentemente y en esta evaluación de PISA 2012, ocupan primeras posiciones por las puntuaciones obtenidas por los estudiantes de Secundaria evaluados, sin embargo se encuentran en últimas posiciones en la evaluación de la práctica docente realizada en TALIS 2013. Países tales como Corea, Japón o Finlandia son un fiel reflejo de las claras discrepancias entre los dos conjuntos de puntuaciones. Igualmente, en sentido inverso, países como Abu Dabi, Rumanía o México, entre otros, se ubican en los últimos lugares en los resultados de PISA 2012, mientras que se sitúan en los primeros lugares en la evaluación de la práctica docente del profesorado. Sin duda, los resultados de este primer análisis nos llevan a reflexionar sobre las posibles causas que pueden subyacer a tal discrepancia y sobre las que nos referiremos, tras las conclusiones que se derivan de los análisis que se realizaron posteriormente.

Las conclusiones derivadas de los análisis realizados con TALIS-PISA Link, donde parece que las relaciones se hubieran debido de producir con más claridad al estar estudiando profesores y alumnos del mismo centro, del mismo nivel educativo y de la misma asignatura (Matemáticas), son similares a las ya mencionadas anteriormente. Países que ocupan posiciones altas en Matemáticas en PISA 2012, obtienen bajas valoraciones en su práctica docente en TALIS 2013. Estas discrepancias se evidencian, tanto cuando se toma la puntuación global de TALIS como 
cuando se toman las distintas dimensiones que configuran el instrumento de práctica docente. Las correlaciones de Pearson calculadas entre estos conjuntos de puntuaciones (Total de práctica docente $\mathrm{y}$ de cada una de sus dimensiones, con la puntuación en Matemáticas PISA 2012) son bajas (aunque significativas, pero irrelevantes por la magnitud de la correlación) y en su mayoría negativas, otra evidencia empírica de la falta de congruencia y de las claras discrepancias entre ambos conjuntos de puntuaciones.

En la misma línea, los análisis realizados para probar las diferencias en Matemáticas en función del distinto nivel de práctica docente (Alto, Medio y Bajo) muestran, igualmente, diferencias significativas e irrelevantes, sin embargo, la puntuación media más alta de los alumnos en Matemáticas se corresponde con los profesores con un nivel bajo en práctica docente, una evidencia más de las conclusiones derivadas de este trabajo.

Estas conclusiones llevan, sin ninguna duda, a plantear algunas reflexiones, interrogantes y cuestionamientos sobre estas evaluaciones que, esperamos, puedan servir para introducir cambios en posteriores ediciones.

En primer lugar, hay que subrayar que ambas evaluaciones no se realizaron para ser objeto de estudio de este tipo, es decir, para relacionar los resultados de ambas, lo cual no invalida, en ningún caso, las evidencias empíricas que muestran las mencionadas discrepancias encontradas. Es evidente que un planteamiento en el diseño de ambas evaluaciones, orientado a un estudio de las relaciones entre los resultados de ambas, posibilitaría llegar a conclusiones integradas y no fragmentadas, con una mayor aplicación práctica para la mejora de las políticas y sistemas educativos, de la formación de profesores, entre otros elementos clave de la educación.

Hay que tener en cuenta, en cualquier caso, las limitaciones de los datos de los que se dispone para realizar el estudio. Por una parte, en los estudios globales de los 32 países, los resultados de PISA corresponden a unos centros $\mathrm{y}$, posiblemente, los resultados de TALIS corresponden a otros, aunque haya una muestra representativa de ambos grupos de cada país, pero es evidente la variabilidad que puede existir entre sus distintos centros. Por otra parte, en los estudios de los 8 países de TALIS-PISA Link, aunque teóricamente la relación parece más clara (profesores y alumnos del mismo centro, mismo nivel educativo y misma asignatura -Matemáticas-), la imposibilidad de emparejar profesor con sus propios alumnos puede generar variabilidad difícil de controlar con los análisis que se pueden realizar.

Pero ¿cuáles son las posibles causas o hipótesis en que se pueden sustentar estos resultados? Por una parte, sería importante analizar la fundamentación teórica que sustenta cada una de las evaluaciones, especialmente la relativa a TALIS, cuyo enfoque pudiera no responder al modelo/s de práctica docente del profesorado de algunos de los países. Igualmente, razones de tipo contextual y cultural pueden estar en la base de estas discrepancias, lo que nos llevaría a tener en cuenta algunas variables relevantes $y$ diferenciadoras entre los países que podrían explicar, en gran parte, las diferencias mostradas.

Por otra parte, aunque existe una gran experiencia en la elaboración de las pruebas de PISA por las ya repetidas ediciones y por los reiterados y exhaustivos estudios técnicos y psicométricos de las mismas, parece que no lo es tanto en los instrumentos utilizados para la evaluación de profesores, los cuales podrían precisar de estudios técnicos y psicométricos que pudieran aportar mayor información respecto a la consistencia, fiabilidad y validez de los mismos.

En síntesis, consideramos de especial relevancia el planteamiento de procesos de elaboración de ambas evaluaciones que puedan ser puestas en relación desde su diseño, analizadas técnicamente con el rigor exigido en los procesos evaluativos $\mathrm{y}$, en 
consecuencia, en los instrumentos. Por otra parte, se precisa impulsar estudios relacionados con cada una de las dos evaluaciones $\mathrm{y}$, especialmente, aquellos que permitan estudiar las relaciones entre los resultados de ambas para incorporar mejoras en todos y cada uno de los países derivados de las conclusiones de los mismos.

\section{REFERENCIAS}

Albergaria-Almeida, P., da Silva Lopes, B., \& Martinho, M. (2015). Student assessment strategies in Portugal: an analysis of TALIS 2013. Procedia-Social and Behavioral Sciences, 186, 841-846. doi: http://dx.doi.org/10.1016/j.sbspro.2015.04.143

Calero, J., \& Escardíbul, J. O. (2012). El rendimiento del alumnado de origen inmigrante en PISA 2012. PISA, 4-31.

Carabaña, J. (2015). La inutilidad de PISA para las escuelas. Madrid: La Catarata.

Clotfelte, C.T., Ladd, H. F. \& Vigdor, J. L. (2007). Teacher Credentials and Student Achievement in High School: A Cross Subject Analysis with Fixed Effects. Journal of Human Resources, 45, 655-681. doi: http://dx.doi.org/10.3386/w13617

Dohn, N. B. (2007). Knowledge and skills for PISA-Assessing the assessment. Journal of Philosophy of Education, 41(1), 1-16. doi: http://dx.doi.org/10.1111/j.14679752.2007.00542.x

Eveleigh, F., \& Freeman, C. (2012). An exploratory analysis of the TALIS and PISA link data: an investigation of the possible relationships. The Need for Educational Research to Champion Freedom, Education and Development for All, ECER 2012.

Fernández-Díaz, M. J., Rodríguez-Mantilla, J. M., \& Martínez-Zarzuelo, A. (2015). Teaching Practice of Secondary Education Teachers in Spain according to TALIS 2013. Revista Española de Pedagogía, 73(261), 225-244.

Ferrer, F. (2012). PISA: Aportaciones e incidencia sobre las políticas educativas nacionales. Revista española de educación comparada,

(19),

11-16.

http://dx.doi.org/10.5944/reec.19.2012.7941

Font, C. M., Badia, M. C., Alemany, I. G., Besora, M. C., Gisbert, D. D., Arce, R. L., Alonso, P. C., Seuba, M. C., Castilla, M. N., Lamo, S. S., Valdivia, I. M. Á., Villanueva, L. V. \& Boekaerts, M. (2009). Pisa como excusa.: Repensar la evaluación para cambiar la enseñanza (Vol. 24). Grao.

Grek, S. (2009). Governing by numbers: The PISA 'effect'in Europe. Journal of education policy, 24(1), 23-37. doi: http://dx.doi.org/10.1080/02680930802412669

Gumus, E., \& Bellibas, M. S. (2016). The effects of professional development activities on principals' perceived instructional leadership practices: multi-country data analysis using TALIS 2013. Educational Studies, 1-15. Doi: http://dx.doi.org/10.1080/03055698.2016.117 $\underline{2958}$

Gustafsson, J. E. (2003). What Do We Know About Effects of School Resources on Educational Results?. Swedish Economic Policy Review, 10, 77-110.

Hair, J., Anderson, R., Tathan, R. \& Black, W. (2009). Análisis multivariante (Madrid, Pearson).

Hernández, F. H. (2006). El informe PISA: una oportunidad para replantear el sentido de aprender en la escuela secundaria. Revista de educación, (1), 357-379.

Jensen, B., Sandoval, A., Knoll, S \& González, E.J. (2012). The Experience of New Teachers: Results from TALIS 2008. OECD Publishing.

Kaplan, D., \& Turner, A. (2012). Statistical Matching of PISA 2009 and TALIS 2008 Data in Iceland, OECD Education Working Papers, 78. Paris: OECD Publishing.

Kelly, D., Nord, C. W., Jenkins, F., Chan, J. Y., \& Kastberg, D. (2013). Performance of US 15-Year-Old Students in Mathematics, Science, and Reading Literacy in an International Context. First Look at PISA 
2012. NCES 2014-024. National Center for Education Statistics.

Lizasoain, L., Tourón, J., \& Sobrino, Á. (2015). La evaluación del profesorado español y el impacto del feedback en las prácticas docentes. Análisis de TALIS 2013. Revista Española de Pedagogía, 73(262), 465-481.

Marina, J.A. (2014). Prólogo. En INEE (Ed.), TALIS 2013. Estudio Internacional de la Enseñanza y el Aprendizaje. Informe español. Análisis secundario. Madrid: Autor.

Martín, E., \& Rizo, F. M. (2009). Avances y desafíos en la evaluación educativa. Organización de Estados Iberoamericanos para la Educación, la Ciencia y la Cultura.

ME, Ministerio de Educación. (2009). TALIS (OCDE) Estudio Internacional sobre la Enseñanza y Aprendizaje. Informe Español. Madrid: Ministerio de Educación, Subdirección General de Documentación y Publicaciones.

MECD, Ministerio de Educación, Cultura y Deporte. (2014a). PISA 2012 Programa para la evaluación internacional de los alumnos. Informe español. Resultados y contexto. Madrid: Ministerio de Educación, Cultura y Deporte.

MECD, Ministerio de Educación, Cultura y Deporte. (2014b). PISA 2012 Competencia Financiera. Informe español. Madrid: Ministerio de Educación, Cultura y Deporte.

MECD, Ministerio de Educación, Cultura y Deporte. (2014c). PISA 2012 Resolución de problemas de la vida real. Resultados de matemáticas y lectura por ordenador. Informe español. Madrid: Ministerio de Educación, Cultura y Deporte.

MECD, Ministerio de Educación, Cultura y Deporte. (2014d). TALIS 2013. Estudio internacional de la enseñanza y el aprendizaje. Informe español. Madrid: Ministerio de Educación, Cultura y Deporte.

Méndez, I. (2015). Prácticas Docentes y Rendimiento Estudiantil: Evidencia a partir de PISA 2012 y TALIS 2013. Conserjería de Educación, Cultura y Turismo del Gobierno de la Rioja, Fundación Santillana e Instituto Nacional de Evaluación Educativa.

Mendizábal, Á. C. (2016). Explorando los límites de PISA. RASE: Revista de la Asociación de Sociología de la Educación, 9(1), 163-165.

Ming-ren, Z. H. A. O. (2015). The Status and Implications of Teachers' Professional Development in An International Perspective: Based on the Results of TALIS 2013. Teacher Education Research, 3, 016.

Mortimore, P. (2009). Alternative models for analysing and representing countries' performance in PISA. Paper commissioned by Education International Research Institute, Brussels, Belgium.

Neves, C. (2008). Las organizaciones internacionales y la evaluación de los sistemas de educación y formación: Análisis crítico y comparativo. Revista Europea de Formación Profesional, (45), 78-98.

OECD, Organisation for Economic Cooperation and Development. (2009). Creating effective teaching and learning environments: First results from TALIS. Paris: OECD Publishing.

OECD, Organisation for Economic Cooperation and Development (2013a). PISA 2012 Results: Excellence through Equity. Giving every student the chance to succeed. Vol II. OECD Publishing.

OECD, Organisation for Economic Cooperation and Development (2013b). PISA 2012 Results: Ready to Learn. Students'engagement, drive and self-beliefs. Vol III. OECD Publishing.

OECD, Organisation for Economic Cooperation and Development (2013c). PISA 2012 Results: What Makes Schools Successful? Resources, policies and practices. Vol IV. OECD Publishing. 
OECD, Organisation for Economic Cooperation and Development (2013d). PISA 2012. Technical Report. OECD Publishing.

OECD, Organisation for Economic Cooperation and Development (2014a). PISA 2012 Results: What Students Know and Can Do. Student performance in mathematics, reading and science. Vol I. OECD Publishing.

OECD, Organisation for Economic Cooperation and Development (2014b). PISA 2012 Results: Creative Problem Solving. Students' skilla in tackling real-life problems. Vol V. OECD Publishing.

OECD, Organisation for Economic Cooperation and Development (2014c). PISA 2012 Results: Students and Money. Financial Literacy skills for the $21^{\text {st }}$ century. Vol VI. OECD Publishing.

OECD, Organisation for Economic Cooperation and Development. (2014d). TALIS 2013 Results: An International Perspective on Teaching and Learning. Paris: OECD Publishing.

Peña-López, I. (2012). PISA 2012 Assessment and Analytical Framework. Mathematics, Reading, Science, Problem Solving and Financial Literacy.

Pérez, A. I., \& Soto, E. (2011). Luces y sombras de PISA. Sentido educativo de las evaluaciones externas. Cultura y Educación, 23(2), 171-182. doi: http://dx.doi.org/10.1174/113564011795944758

Perry, S. M., Sealy, K. M., \& Hawkins, R. J. (2016). Opportunity to Learn and Students from Economically Disadvantages Homes: Implications for School Leaders Through an Examination of TALIS 2013. Available at SSRN (Social Science Research Network) 2717054.

Ruiz, J. B., Gil, M. G., Navas, M. F., Ramos, R. Y., Ruiz, M. P. S., \& Núñez, M. J. S. (2011). Todos queremos ser Finlandia”. Los efectos secundarios de PISA. Education in the Knowledge Society (EKS), 12(1), 320339.
Sánchez, C. C., \& Delgado, F. J. H. (2013). Informe PISA en España. Un análisis al detalle. Profesorado. Revista de Currículum y Formación del Profesorado, 17(2), 20.

Sánchez, E. \& García-Rodicio, H. (2006). Relectura del estudio PISA: qué y cómo se evalúa e interpreta el rendimiento de los alumnos en la lectura. Revista de educación, (1), 195-226.

Sealy, K. M., Perry, S. M., \& DeNicola, T. C. (2016). Relationships and Predictors of Principal Job Satisfaction Across Multiple Countries: A Study Using Talis 2013 and PISA 2012. Available at SSRN 2717056.

Sedghi, A., Arnett, G., \& Chalabi, M. (2013). Pisa 2012 results: which country does best at reading, maths and science. The Guardian. Retrieved October, 21, 2015.

Sjøberg, S. (2015). PISA and global educational governance-A critique of the project, its uses and implications. Eurasia Journal of Mathematics, Science \& Technology Education, 11(1), 111-127. doi http://dx.doi.org/10.12973/eurasia.2015.1310a

Stacey, K. (2015). The international assessment of mathematical literacy: PISA 2012 framework and items. In Selected Regular Lectures from the 12th International Congress on Mathematical Education (pp. 771-790). Springer International Publishing.

Thomson, S., De Bortoli, L., \& Buckley, S. (2013). PISA 2012: How Australia measures up. Melbourne: Australian Council for Educational Research.

Vieluf, S., Kaplan, D., Klieme, E. \& Bayer, S. (2012). Teaching Practices and Pedagogical Innovation: Evidence from TALIS. OECD Publishing.

Villar, A. (2013). Rendimiento, esfuerzo y productividad: análisis de los resultados en matemáticas de los estudiantes españoles según PISA (2012). PISA 2012: Programa para la evaluación internacional de los alumnos. 
Wayne, A. J. \& Youngs, P. (2003). Teacher characteristics and student achievement gains: A review. Review of Educational Research, 73, 89-122.

Wenglinsky, H. (2002). How schools matter: The link between teacher classroom practices and student academic performance. Education Policy Analysis Archives, 11(12).

Wheater, R. (2013). Achievement of 15 year olds in England: PISA 2012 national report (OECD Programme for International Student Assessment), December 2013.

\section{ANEXO}

Estudios descriptivos de TALIS 2013 ordenados por la puntuación global obtenida en el Ítem Total

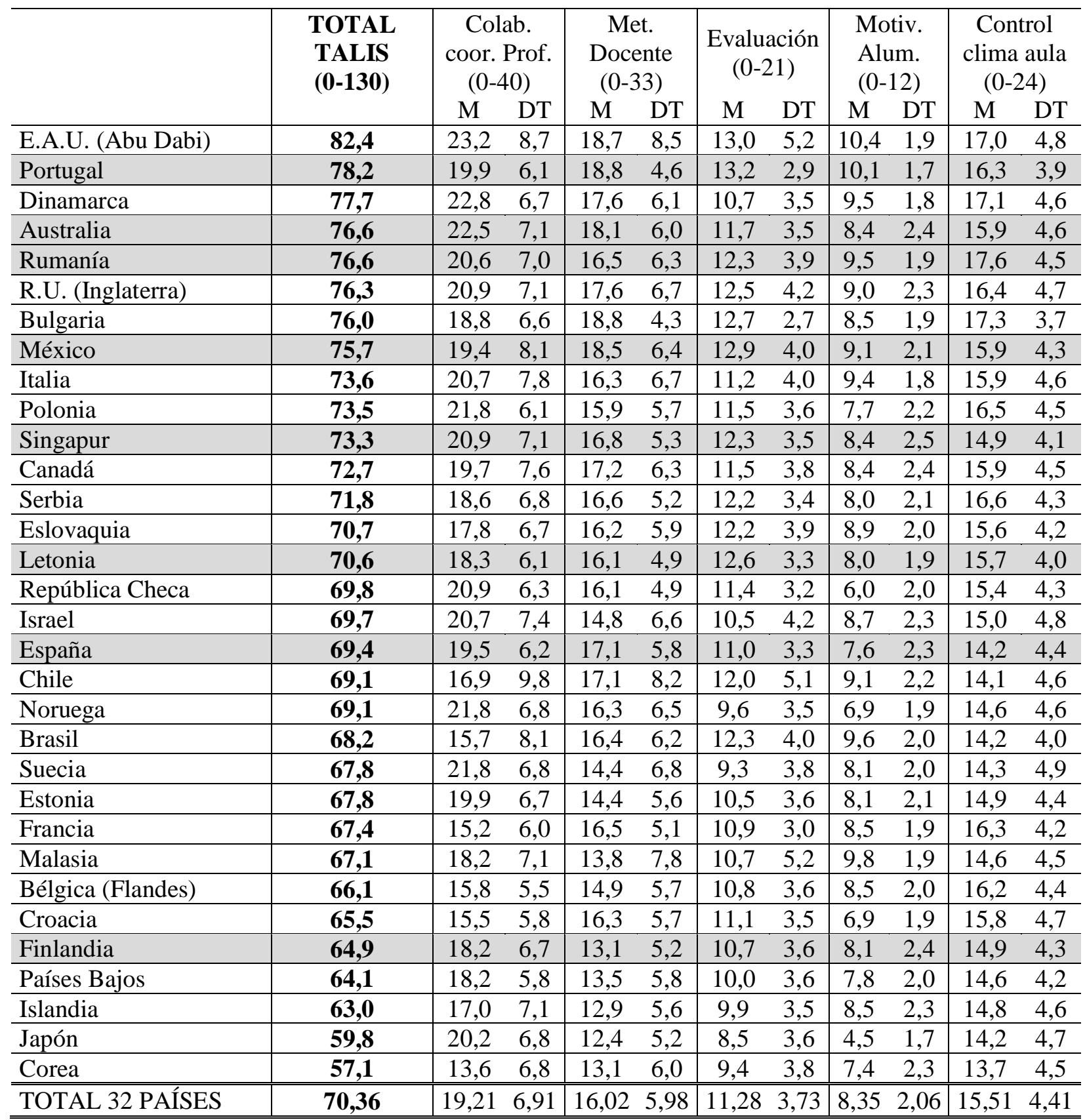




\section{Authors / Autores}

To know more / Saber más

María-José José Fernández-Díaz mjfdiaz@edu.ucm.es

Decana de la Facultad de Educación de la Universidad Complutense de Madrid y Profesora del Departamento de Métodos de Investigación y Diagnóstico en Educación. Sus principales áreas de especialización son: metodología de investigación, evaluación y medida en educación, dirección y liderazgo, formación de profesores y calidad y evaluación de centros, profesores y programas educativos. Colabora con universidades extranjeras y otros organismos internacionales

\section{Rodríguez-Mantilla, Jesús-Miguel jesusmro@ucm.es}

Doctor en Educación, Licenciado en Psicopedagogía y Diplomado en Magisterio de Educación Primaria por la Universidad Complutense de Madrid, es profesor de Métodos de Investigación en la Universidad Complutense de Madrid y en la Universidad Camilo José Cela. Sus últimos trabajos y publicaciones se enmarcan en el estudio del síndrome de Burnout, Evaluación de Competencias docentes, Clima

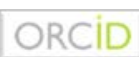

$\underline{0000-0002-0964-4342}$

\section{ResearchGate} escolar y en el análisis de la Calidad de las instituciones educativas.

\section{Martínez-Zarzuelo, Angélica angelica.martinez@ucm.es}

Doctora en Educación por la Universidad Complutense de Madrid. Licenciada en Matemáticas por la Universidad de Valladolid, con Máster Universitario en Investigación Matemática por la Universidad Complutense de Madrid. Con experiencia en evaluaciones externas nacionales e internacionales del sistema educativo en sus diferentes fases: muestreo, aplicación, seguimiento, codificación, análisis de datos y realización de informes, en el marco del Instituto Nacional de Evaluación Educativa

\section{RELIEVE}

\section{Revista ELectrónica de Investigación y $\mathbf{E V}$ aluación Educativa E-Journal of Educational Research, Assessment and Evaluation \\ [ISSN: 1134-4032]}

(C) Copyright, RELIEVE. Reproduction and distribution of this articles it is authorized if the content is no modified and their origin is indicated (RELIEVE Journal, volume, number and electronic address of the document).

(C) Copyright, RELIEVE. Se autoriza la reproducción y distribución de este artículo siempre que no se modifique el contenido y se indique su origen (RELIEVE, volumen, número y dirección electrónica del documento). 